INTRODUCTION

\title{
NUMÉRO SPÉCIAL INTRODUCTION LES JEUNES CONTREVENANTS
ET CONTREVENANTES:
TROUVER UN JUSTE ÉQUILIBRE
ENTRE CONTRÔLE ET TRAITEMENT
}

\author{
PRUE RAINS \\ Université McGill \\ ELI TERAM \\ Université Wilfrid-Laurier \\ et \\ CÉCILE TOUTANT \\ Institut Philippe Pinel de Montréal
}

\begin{abstract}
La réaction du Québec aux changements proposés au cadre législatif canadien à l'égard des jeunes contrevenants et contrevenantes met en lumière plusieurs des interrogations soulevées dans ce numéro spécial. Dès le dépôt du projet de Loi sur le système de justice pénale pour les adolescents (LSJPA) (Canada, 2001) par Anne McLellan, alors ministre fédérale de la justice, les intervenantes et intervenants professionnels du Québec ont exprimé leur opposition au remplacement de la Loi sur les jeunes contrevenants (LJC) (Canada, 1982/1985). Selon eux, une nouvelle loi est inutile puisque les faiblesses de la LJC ne relèvent pas de la loi elle-même mais bien de son application, tel que démontré dans le rapport Jasmin (Québec, 1995). Au lieu d'adopter une nouvelle loi, les gouvernements feraient mieux d'affecter les dépenses à d'autres secteurs comme l'éducation publique et le développement de nouvelles ressources, aux deux extrémités du continuum d'intervention auprès des jeunes.
\end{abstract}

La Coalition pour la justice des mineurs, qui regroupe des représentants et représentantes du monde de la justice, du travail social, du milieu universitaire, des services policiers et des syndicats du Québec et du reste du Canada, a fourni une critique serrée de la Loi C-7, alléguant qu'en dépit des modifications apportées par la ministre McLellan, la loi brise l'équilibre des principes qui faisaient le succès de la LJC: il faut bien entendu des conséquences aux gestes commis mais il est nécessaire de maintenir des modalités de responsabilisation et de réadaptation adaptées aux jeunes. La Loi C-7 met trop d'emphase sur l'infraction, aux dépens d'une bonne évaluation des besoins des jeunes et des mesures correctives nécessaires à leur développement. La nouvelle loi créé deux vastes catégories de jeunes contrevenants et contrevenantes, basées sur la sévérité des infractions: les infractions mineures (traitées dans la communauté) et les infractions majeures (passibles de peines adultes). Cette façon de traiter les jeunes contrevenants et contrevenantes marque une régression claire par rapport aux pratiques actuellement en vigueur au 
Québec, qui tiennent compte à la fois de l'infraction et des circonstances de vie particulières à l'individu concerné. Selon la coalition:

$\mathrm{Au}$ lieu de gaspiller les fonds publics à implanter un nouveau système dont on n'a pas besoin, le gouvernement se montrerait un gestionnaire avisé en investissant ces sommes dans le renforcement de l'actuelle Loi sur les jeunes contrevenants. Ce faisant, il répondrait aux vœux des chercheurs et des intervenants qui travaillent auprès des jeunes et qui font consensus pour affirmer que ce n'est pas la Loi sur les jeunes contrevenants qui doit être remplacée, mais le public qui doit être mieux informé, les jeunes et leurs communautés qui doivent être mieux soudés.

Il ne fait aucun doute que la nouvelle loi, qui est le fruit de motivations politiques, continuera à soulever bien des litiges entre le gouvernement fédéral et certaines provinces. Considérant l'aspect discrétionnaire de certains des règlements de la LSJPA, il est même possible que son application donne lieu à des pratiques contradictoires. D'une part, une province comme le Québec pourrait choisir de continuer à privilégier le traitement et la réadaptation; de même, l'augmentation du financement pourrait inciter d'autres provinces à mettre sur pied des mesures extrajudiciaires basées sur la communauté. D'autre part, en conséquence de la Loi, les sentences pourraient par contre devenir plus sévères, les jeunes plus largement considérés comme adultes et leur image de «prédateurs », davantage renforcée.

On ne peut prévoir la façon dont la nouvelle loi sera implantée. Cependant, l'orientation choisie par les différentes juridictions en cause sera vraisemblablement inspirée des mêmes considérations politiques qui ont initialement motivé la création de la LSJPA. Chacun à leur manière, les articles réunis ici présentent certains des défis, des possibilités et des contraintes auxquels sont confrontées les personnes qui cherchent à trouver un juste équilibre entre traitement et contrôle pour les jeunes contrevenants et contrevenantes.

Dans le portrait impressionniste qu'il dresse du système canadien de justice pour les jeunes, Jim Hackler dit maintenant regretter d'avoir accordé son soutien à ce qu'on pourrait appeler la « légifération » du système par l'entrée en vigueur de la Loi sur les jeunes contrevenants en 1984. En tant que libéral, il avait milité en faveur du remplacement du tribunal « cas par cas » par des procédures judiciaires formelles, mais il n'avait pas prévu que les mesures de protection accordées par la loi mèneraient à la «punition par délai », ni que l'emphase mise sur la procédure prendrait la place des véritables mesures de soutien à la famille. Ce domaine, qui était autrefois le fief des intervenants et intervenantes en travail social, s'est vu envahir par les représentants et représentantes de la justice et des établissements de garde fermée. Par contraste, Hackler admire les arrangements plus flexibles en cours dans certains pays européens, où les tribunaux sont moins encombrés par des pratiques hostiles et se préoccupent davantage d'apporter un véritable soutien aux jeunes et à leur famille. Il envisage cependant quelques lueurs d'espoir au Canada, où des «bonnes gens » (avocats et avocates, procureurs et procureures, juges et intervenants et intervenantes en travail social) travaillent en collaboration pour définir des manières innovatrices de faire face aux problèmes des jeunes.

La LSJPA pourrait fournir à ces gens de nouvelles occasions de mener à bien leur entreprise. Toutefois, le financement requis pour initier de nouveaux programmes de mesures extrajudiciaires n'est pas garanti et demeure tributaire de la volonté de participation des provinces. De plus, de telles possibilités se limitent aux 


\section{INTRODUCTION}

offenses mineures. En réduisant la gamme d'options pour les coupables d'offenses majeures, la Loi fait fi des réalités sociales qui mènent à la fois à des offenses mineures et majeures. En mettant l'emphase sur le crime plutôt que sur le coupable, la Loi pourrait aussi contribuer à faire oublier que certains contrevenants et contrevenantes mineurs nécessitent des interventions majeures, contrairement à certains contrevenants et contrevenantes majeurs. Tel que démontré par Michael Ungar, Eli Teram et John Picketts, les jeunes trouvés coupables d'offenses majeures ont peutêtre autant besoin d'établir un lien avec leur communauté que les contrevenantes et contrevenants mineurs, voire davantage. En tirant parti de la «sagesse locale » que Hackler a apprise dans d'autres pays et dans certaines communautés canadiennes, nous devrions privilégier les résultats plutôt que la culpabilité.

Rick Prashaw propose un changement similaire dans son article sur la justice réparatrice. À l'instar d'Hackler, il constate les lacunes de l'approche " crime et châtiment ", adoptée par le système pénal formel, lorsqu'il est temps de bâtir une relation entre les jeunes et la communauté. Par l'usage d'histoires recueillies dans tout le Canada, il témoigne éloquemment du pouvoir de la communauté dont les membres se regroupent pour guérir le mal que se font entre eux les individus et pour soigner les relations meurtries. Au lieu de prêcher la responsabilisation par le biais des étrangers et étrangères, la justice réparatrice reconnaît que le message sera transmis beaucoup plus efficacement s'il provient des membres de la commu-nauté qui entretiennent des relations significatives avec les jeunes en question. Plus important encore, le modèle de justice réparatrice contribue à briser les stéréotypes que les gens entretiennent envers les autres. Les jeunes et les membres de la communauté, en exprimant leur attachement mutuel dans un environnement sécuritaire et respectueux, peuvent se rejoindre et commencer à bâtir une relation ou réparer celle qui a été brisée.

En vertu de la LJC, il était possible d'appliquer une approche de justice réparatrice comme mesure extrajudiciaire. Considérant l'emphase accordée par la justice réparatrice à la modification des stéréotypes, il est fort dommage de consta-ter que les coupables d'offenses sérieuses auront dorénavant moins d'occasions de renouer avec la communauté. En faisant de l'offense le point central de l'interven-tion, la nouvelle loi ne donne pas pleine considération aux interventions effectuées en fonction des circonstances sociales et personnelles qui forment le contexte des offenses juvéniles. Trois articles visent à démontrer l'envergure de cette décontextualisation.

L'article signé par Ungar, Teram et Picketts raconte en détail la carrière organisationnelle de deux jeunes nommés Paul et Cameron, qui se retrouvent dans le même établissement pour jeunes contrevenants. L'histoire de ces deux garçons donne un visage humain à un groupe dont la population conserve une image diabolique. Comme bien des jeunes qui traversent le système de sécurité sociale pour enfants et le système de santé mentale, ils ont eu une enfance extrêmement difficile. Toutefois, malgré le fait que leur « désavantage accumulé " ait été à peu près égal, seul Cameron était demeuré en contact avec sa communauté et sa famille, qui ont plaidé sa cause et pris soin de lui lorsqu'il a été placé en institution. Paul, dont la vie malheureuse s'est terminée par un accident de voiture, vivait en étranger dans la communauté, où il était considéré comme un criminel. Le contraste entre les 
deux cas démontre l'impact que la communauté peut avoir, du fait qu'elle tient compte ou ignore le contexte de vie des jeunes contrevenants et contrevenantes.

Anne Cummings et Alan Leschied, pour leur part, décrivent les réalités et le passé d'abus qui mènent aux comportements agressifs chez les adolescentes. Ils ne réduisent pas la violence à une défaillance psychologique mais s'attachent plutôt à démontrer comment les adolescentes perçoivent et expliquent leurs affrontements verbaux et physiques dans le contexte plus large de leurs relations sociales. Ils réclament des programmes de prévention pour aider les adolescentes à trouver des façons plus appropriées de faire face aux conflits et frustrations. Cummings et Leschied nous enjoignent d'intégrer à ces programmes des modules de traitement pour les victimes d'abus, en plus de s'attaquer aux problèmes d'intégrité et de vulnérabilité qui sont fréquemment associés aux enfances turbulentes. Afin de véritablement répondre aux besoins complexes et multidimensionnels des adolescentes, il sera nécessaire d'assurer la concertation entre le système de sécurité sociale pour enfants, le système de santé mentale juvénile et le système de justice.

L'article de Charlotte Waddell, Jonathan Lomas, Dan Offord et Mita Giacomini vise à attirer l'attention sur les jeunes contrevenants et contrevenantes qui présentent des désordres de conduite, ainsi que sur l'écart entre ce que nous savons de ces jeunes, d'une part, et d'autre part les politiques et programmes mis sur pied pour répondre à leurs besoins. Les auteurs et auteures passent en revue les résultats de recherches, ce qui permet de mettre en lumière les contextes fami-liaux dysfonctionnels au sein desquels naissent les désordres de conduite et d'évaluer l'efficacité de diverses interventions. Ils rapportent que les mesures sévères et punitives demeurent inefficaces, que l'incarcération empire probable-ment les problèmes et enfin que les interventions primaires et secondaires plus larges sont plus efficaces. Dans la mesure où la fréquence des désordres de con-duite est élevée chez les jeunes qui sont coupables d'offenses majeures, il demeure inutile de réagir en fonction de leurs offenses plutôt qu'en fonction de leurs pro-blèmes.

L'article signé par Francine Julien-Gauthier, Marc Daigle et Line Bellemare propose une solution alternative à ces approches vaines et décontextualisantes. Les auteurs et auteures décrivent un programme d'aide pour les jeunes de 10 et 11 ans qui en sont à leur première offense. Par le biais d'une métaphore où le point final se change en virgule, le programme considère la première offense comme une er-reur de parcours et une occasion de changement, au lieu d'un échec fatal. Ils soutiennent que « les programmes novateurs ne doivent pas leur succès qu'à leur originalité ou leur pertinence. Ils doivent aussi s'élaborer à partir d'un consensus dans la communauté, de l'expertise et de la crédibilité du personnel et d'une approche évaluative. " Les gens qui sont sceptiques face aux chances de succès des interventions auprès des très jeunes contrevenants et contrevenantes pourraient trouver dans ce programme un modèle utile.

Quatre articles examinent la façon dont les instances juridiques et institutionnelles traitent les jeunes contrevenants et contrevenantes. Brian Howe et Katherine Covell se servent des obligations du Canada en vertu de la Convention des nations unies sur les droits de l'enfant comme points de référence pour évaluer la Loi sur le système de justice pénale pour les adolescents par rapport à la LJC. Leur verdict est mitigé. D'une part, ils constatent que la LSJPA s'inscrit dans la perspective de la convention en raison de l'emphase accordé au traitement, à la réadaptation et à la 


\section{INTRODUCTION}

réinsertion, ainsi que le recours restreint à la mise sous garde. D'autre part, ils sont préoccupés par certains autres éléments, comme la possibilité toujours existante de mêler les jeunes aux adultes, la clause de publication du nom des jeunes contrevenants et contrevenantes et les options élargies d'imposer des peines pour adultes. De plus, même les aspects prometteurs de la loi sont compromis par leur nature discrétionnaire. (De fait, l'un de nos analystes observait que contraire-ment aux améliorations essentiellement discrétionnaires que comporte la LSJPA, les détériorations comme les sentences adultes et la publication des noms demeurent significatives et moins discrétionnaires). Howe et Covell concluent en recommandant d'instaurer un mécanisme de contrôle obligatoire du traitement réservé aux jeunes contrevenants et contrevenantes dans les établissements de détention et de garde, qui serait exercé par des organismes indépendants comme les bureaux de défense des droits des enfants.

L'article de Carla Cesaroni prend la relève sur ce point, en présentant des preuves convaincantes de la nécessité d'établir des normes pour régir les établissements de garde. Considérant l'usage répandu de la garde au Canada ainsi que les abus fort documentés dont les jeunes ont été victimes en institution, l'absence de telles normes est surprenante. Les jeunes placés en milieu de garde sont vulnérables et ont plusieurs besoins spéciaux. Or il semble que certains établissements négligent de répondre à des besoins élémentaires. Cesaroni identifie des carences relatives à la nourriture, aux soins de santé, à l'éducation, aux programmes et à l'environnement physique. D'autres préoccupations concernent la limitation des contacts avec la famille et les amis et amies, ou le manque de sensibilité à la culture et au sexe de l'individu. Même si la LSJPA apporte des changements im-portants au plan de la garde, la plupart sont limités à l'attribution de sentence et à la réinsertion. Il est clair que l'établissement de normes visant les soins de garde devrait constituer une priorité pour les responsables des politiques.

Les programmes résidentiels comme ceux que Bruce Bidgood et Mark Pancer ont étudiés pourraient peut-être donner de meilleurs résultats s'ils étaient régis par des normes clairement définies. Dans le cadre de cette étude, les auteurs évaluent six programmes. Ils soulignent le potentiel des programmes quant au succès des interventions et identifient certains éléments des programmes qui sont jugés les plus utiles par les contrevenants et contrevenantes, notamment les relations établies avec le personnel à plein temps ainsi que les activités récréatives à l'interne et à l'externe. Étant donné le large recours à la mise sous garde dans le système actuel, il est important de continuer à tenir compte de l'expérience des jeunes et de leur famille face aux interventions réalisées durant ces placements. Sans cette contribution, les milieux de garde sont passibles de devenir de simples endroits de confinement, sans autre objectif.

De plus, étant donné que la LSJPA pourrait élargir davantage le recours à la garde, il importe d'analyser l'utilisation efficace des établissements. À cet égard, Ungar, Teram et Picketts proposent un nouveau cadre d'analyse. Ils soutiennent que les établissements, afin d'être efficaces, doivent travailler étroitement avec la communauté pour accueillir les jeunes contrevenants et contrevenantes à risque élevé. Leur suggestion consiste à resituer les établissements pour en faire des prolongements de la communauté et à les utiliser stratégiquement pour contenir les jeunes contrevenants et contrevenantes tel que requis. Ainsi, les membres de la 
communauté et les intervenants et intervenantes en travail social ne se désengageraient pas des jeunes à risque élevé en milieu de garde, mais entreprendraient plutôt de planifier leur réinsertion dans la communauté dès que ceux-ci seraient placés en institution. Même si la LSJPA mentionne spécifiquement les plans de réadaptation et de réinsertion, on n'indique pas qui sera responsable d'élaborer ces plans. Le fait de resituer les établissements aiderait à réduire la dichotomie entre les jeunes dans la communauté et les jeunes en milieu de garde.

Les articles réunis ici touchent à plusieurs questions reliées à l'équilibre entre contrôle et traitement pour les jeunes contrevenants et contrevenantes. Si l'on ne connaît pas l'historique de la législation relative à la justice pour les jeunes et les motivations politiques dont elle est issue, on pourrait croire que la LSJPA présente une approche équilibrée. Cependant, on ne peut ignorer le fait que les politiques gouvernementales sont développées et implantées dans des contextes sociaux particuliers. Cette loi a vu le jour dans le contexte des protestations du public à l'endroit de la présumée indulgence excessive de la Loi sur les jeunes contrevenants, suite à certains rapports sensationnalistes de violence juvénile. En résultat, la nouvelle loi tente de satisfaire aux attentes de tous: termes sévères pour apaiser ceux qui demandent de sévir, et langage plus tempéré pour les défenseurs de la prévention et de l'intervention communautaire en équilibre symbolique. Reste à savoir lequel de ces deux registres prévaudra, mais l'histoire ne peut que nous dicter un certain pessimisme. Considérant l'actuelle école de pensée, il sera sans doute plus facile, comme par le passé, de trouver les fonds publics pour les aspects plus « durs » du système, alors que les aspects plus « doux » devront se contenter de ressources limitées (strictement rien, pour être franc). Il est certain que les décisions prises par les joueurs clés (juges, procureurs et procureures, intervenants et intervenantes auprès des jeunes) seront guidées par les ressources disponibles et par les attitudes culturelles dominantes envers les jeunes contrevenantes et contrevenants. Selon toute probabilité, il sera plus aisé pour les gens qui prennent ces décisions de réagir aux offenses plutôt qu'à leur contexte et de favoriser le contrôle au lieu du traitement.

\section{RÉFÉRENCES}

Canada. (1982/1985). Loi sur les jeunes contrevenants. L.R.C. 1985, Chap. Y-1.

Canada. (2001). 37ième Parlement. Loi C-7, Loi sur le système de justice pénale pour les adolescents.

Québec. (1995). Au nom . . . et au-delà de la loi. Rapport du Groupe de travail chargé d'étudier l'application de la Loi sur les jeunes contrevenants au Québec (Rapport Jasmin). Québec, QC: Ministère de la justice et Ministère de la santé et des services sociaux. 